\title{
Gobierno corporativo y desempeño en las universidades chilenas
}

\author{
Sergio Zuniga-Jara ${ }^{1 *}$ y Oscar Sjoberg-Tapia ${ }^{2}$ \\ (1) Escuela de Ciencias Empresariales, Univ. Católica del Norte, Larrondo 1281 Coquimbo, Chile (correo-e: sz@ucn.cl). \\ (2) Facultad de Ciencias Empresariales, Univ. Arturo Prat, Avda. Arturo Prat 2120, Iquique, Chile \\ (correo-e: osjoberg@unap.cl). \\ * Autor a quien debe ser enviada la correspondencia
}

Recibido Ene. 5, 2020; Aceptado Mar. 3, 2020; Versión final May. 10, 2020, Publicado Feb. 2021

\begin{abstract}
Resumen
El objetivo de esta investigación es analizar en qué medida el desempeño académico y económico de las universidades chilenas se relaciona con su modelo de gobierno corporativo. Se utilizaron dos pruebas no paramétricas (chi-cuadrado de Pearson y likelihood-ratio) de independencia condicional. Los resultados muestran que el desempeño académico aparece asociado estadísticamente con el tipo de universidad (privadas y estatales). Las universidades estatales presentan las mayores caídas en los rankings académicos (SClmago 2009-2018). Las universidades que usan votación directa registran mayores caídas en ranking, y las universidades mejor clasificadas (privadas y estatales) tienden a designar rectores con un perfil de investigador destacado. Los resultados financieros positivos de las universidades (creación de valor) están correlacionados directa y significativamente con rectores designados por comités externos, y no vía votación directa de los académicos. Se concluye que el desempeño de las universidades chilenas está asociado con la forma en como los rectores son designados.
\end{abstract}

Palabras clave: universidad; gobierno corporativo; valor económico agregado

\section{Corporate governance and performance in Chilean universities}

\begin{abstract}
The primary objective of this research study is to examine whether academic and economic performance of Chilean universities is related to their corporate governance model. Two nonparametric tests (Pearson's chisquare and likelihood-ratio) of conditional independence are used. The results show that academic performance is statistically associated with university type (public and private). Public universities show the greatest drops in academic rankings (SClmago 2009-2018). Universities that use direct voting to select their presidents showed the greatest drops in university rankings. The best-ranked universities (public and private) tend to appoint presidents that have outstanding research profiles. Positive financial university outcomes (value creation) are directly and significantly correlated with presidents appointed by external committees (boards), and not via direct voting by academics. It is concluded that university performance is associated with how university presidents are appointed.
\end{abstract}

Keywords: university; corporate governance; economic added value 


\section{INTRODUCCIÓN}

El desempeño de las universidades constituye un foco de interés para un gran número de agentes, incluyendo estudiantes, académicos, el gobierno, y eventualmente para toda la sociedad. Actualmente no existe una medida ampliamente aceptada de desempeño de las universidades, y suele utilizarse rankings como aproximaciones, junto a varios sistemas de acreditación internacional (Frenken, et al. 2017 y Reyes, 2016). La aparición de universidades privadas en Chile a partir de 1980 ha generado una dinámica importante en el sistema. Esto es muy diferente a la relativa estabilidad en las posiciones de desempeño de las 8 universidades que existían hasta esa fecha. Actualmente en Chile existen formalmente 61 universidades, las que se pueden clasificar como sigue: 16 universidades estatales, 9 universidades privadas tradicionales (creadas antes de 1980 o sus derivadas, Red-G9), y 34 universidades privadas nuevas (creadas después de la reforma universitaria de 1980).

En años recientes (2009-2018) la dinámica en las posiciones en los rankings internacionales parece afectar con mayor fuerza a la baja a las universidades estatales chilenas en comparación con las nuevas universidades privadas (Tabla 1). La Tabla 1 muestra también estimaciones del Valor Económico Agregado (Economic Value Added, EVA) para cada universidad (Zuniga-Jara et al., 2020), sugiriendo un desempeño económico inferior en las universidades estatales. Esto alerta de una potencial crisis en el sector, señalada por Bernasconi (2008b). También ha hecho surgir críticas al sistema de gobierno universitario, es decir a la forma en las cual estas instituciones formalmente se organizan y gestionan (Edwards, 2018; Brunner, 2011; Nahuelhual, 2011). Por ello, la hipótesis central de nuestro estudio es que en las universidades chilenas existe un vínculo relevante entre el tipo de gobierno universitario y el desempeño (académico y/o económico). Las características del sistema universitario en Chile favorecen este tipo de análisis, por cuanto conviven universidades tradicionales (públicas y privadas), con las nuevas universidades privadas. Este estudio forma parte de uno más amplio, para evaluar la hipótesis de la relación gobierno-desempeño, pero a nivel internacional (Aghion, et al., 2010).

Tabla 1. Medidas de Desempeño de las Universidades Chilenas. Cálculos a partir del Ranking Scimago, CGR (2016) y estados financieros de www.mifuturo.cl.

\begin{tabular}{|l|l|l|l|l|}
\cline { 3 - 4 } \multicolumn{2}{c|}{} & $\begin{array}{l}\text { Universidades } \\
\text { Estatales }\end{array}$ & $\begin{array}{l}\text { Universidades } \\
\text { Red G9 }\end{array}$ & $\begin{array}{l}\text { Universidades } \\
\text { Privadas (no G9) }\end{array}$ \\
\hline $\begin{array}{l}\text { Tendencias en el ranking Scimago } \\
(2009-2018)\end{array}$ & Caídas en el ranking & 8 & 5 & 4 \\
\cline { 2 - 5 } & Mejoras en el ranking & 2 & 2 & 4 \\
\hline $\begin{array}{l}\text { Aportes Directos del Estado de Chile } \\
\text { promedio por universidad } \\
\text { (millones de pesos chilenos) }\end{array}$ & A universidades & $\$ 16.614$ & $\$ 21.348$ & $\$ 550$ \\
\cline { 2 - 5 } & A estudiantes & $\$ 15.697$ & $\$ 24.196$ & $\$ 10.353$ \\
\hline $\begin{array}{l}\text { Economic Value Added, EVA (2017) } \\
\text { (millones de pesos chilenos) }\end{array}$ & Promedio & $\$-3.492$ & $\$ 5.080$ & $\$ 2.255$ \\
\cline { 2 - 5 } & Mediana & $\$-1.107$ & $\$ 4.518$ & $\$ 851$ \\
\hline
\end{tabular}

\section{Sistemas de gobierno universitarios en el mundo}

Categorizar los sistemas de gobierno universitarios en el mundo es una tarea difícil (Bernasconi, 2018). A pesar de ello, una clasificación útil es la de Brunner (2011, su diagrama I), sobre la cual puede proponerse una evolución gradual en el tiempo desde modelos burocráticos prusianos y franceses, a los modelos colegiales, modelos de partes interesadas, para dirigirse a esquemas de tipo emprendedor. Nosotros usamos una medida simple como proxy de tipo de gobernanza: la forma en que es elegido el rector y su perfil profesional. En base a esto, hemos categorizado los sistemas corporativos universitarios chilenos entre dos extremos opuestos: los autogestionados-colegiados y los corporativos.

En un sistema colegiado puro, las autoridades administrativas de la universidad se determinan por voto directo de los mismos profesores (se vota directamente por el nombre de un candidato a rector, decano o director de departamento), participando eventualmente como votantes otros trabajadores de la universidad y los estudiantes. Este es el sistema que predomina, en lo general, en los países latinoamericanos, España e Italia (Ordorika, 2015). En este enfoque se critica la surgencia de endogamia, corporativismo, asambleísmo y participación de legos (no expertos) en decisiones técnicas, elementos que parecieran llevar a las universidades a una situación de crisis (Bernasconi, 2008a). Rodríguez (2016) señala que este modelo ha conducido a la desnaturalización de la función educativa y académica de la universidad, con una creciente desconexión con el medio, magnificación de conflictos internos, y abandono de la misión institucional, entre otros. Respecto de las universidades estatales chilenas, también se ha criticado que el gobierno universitario no ha sido objeto de iniciativas de reforma (Torres, 2016). Este modelo generaría universidades capturadas por sus académicos, con carreras muy largas, gran burocracia y mallas curriculares sobrecargadas. Brunner y Peña (2008) discuten el poder de los académicos por sobre grupos externos y otros miembros de la 
comunidad universitaria. Beyer y Paredes (Nahuelhual, 2011) indican que en las universidades estatales chilenas se debiera implementar un consejo directivo externo, restringiendo la participación académica a aspectos específicos, mejorar la transparencia (accountability) y la cautela de los intereses del Estado.

En un sistema corporativo puro, las autoridades de la universidad son designadas por alguna junta directiva, sin que exista participación directa de profesores o estudiantes. Este modelo predomina en los Estados Unidos y en las universidades mejor rankeadas en el mundo (Tabla 2). Edwards (2018) señala que en ninguna universidad estadounidense hay votaciones para la elección de las autoridades, lo que no habría afectado la convivencia ni el desempeño. Se considera que el rector debe ser seleccionado con criterios técnicos por grupos de expertos, observando practicas exitosas en empresas privadas (Henze, 2010, y UWN, 2016). Lo más común es que un nuevo rector sea un directivo de otra institución, e incluso de otro país (Bleiklie y Kogan, 2007; Paradeise et al. 2009). El proceso de selección e inducción suele ser exigente, con perfiles de competencias y programas de formación especial a ser completados por un nuevo rector (Rupp et al., 2016). En la experiencia internacional, este esquema también perece terminar con el problema de los compromisos adquiridos por los rectores-candidatos con grupos internos.

La Tabla 2 muestra que a nivel de los países de la OECD coexiste diversidad de modelos de gobierno universitario, bajo los criterios explicitados anteriormente. Una observación importante de esta tabla es que las mejores universidades del mundo (las mejores rankeadas) generalmente pertenecen a los países con sistemas de gobierno universitario de tipo corporativo. Como veremos, esta idea es revisada en el caso chileno en la Hipótesis 1 de este estudio. Es interesante notar aquí el caso de la Universidad de Cambridge, en que hasta 1992 el vice-chancellor (rector) era elegido anualmente entre los jefes de los Colegios de Cambridge, y que ahora es designado por el Council entre un grupo internacional de candidatos (en UK el "Chancellor" no es el rector, sino un puesto ceremonial).

Tabla 2. Sistema de Gobierno en las Tres Mejores Universidades de Cada País de la OECD. Usando como proxy la forma de elección del rector. Elaboración de los autores.

\begin{tabular}{|c|c|c|c|c|c|}
\hline País & Localización & Predominante & País & Localización & Predominante \\
\hline Austria & Europa & Corporativo & España & Europa & Democrático \\
\hline Bélgica & Europa & Democrático & Suecia & Europa & Corporativo \\
\hline Republica Checa & Europa & Democrático & Suiza & Europa & Corporativo \\
\hline Dinamarca & Europa & Corporativo & Reino Unido & Europa & Corporativo \\
\hline Estonia & Europa & Mixto & Israel & Asia & Mixto \\
\hline Finlandia & Europa & Corporativo & Japón & Asia & Democrático \\
\hline France & Europa & Corporativo & South Corea & Asia & Corporativo \\
\hline Alemania & Europa & Corporativo & Turquía & Asia & Mixto \\
\hline Grecia & Europa & Democrático & Canadá & Norte América & Corporativo \\
\hline Hungría & Europa & Mixto & México & Norte América & Mixto \\
\hline Islandia & Europa & Mixto & Estados Unidos & North América & Corporativo \\
\hline Irlanda & Europa & Mixto & Australia & Oceanía & Corporativo \\
\hline Italia & Europa & Democrático & New Zelanda & Oceanía & Corporativo \\
\hline Letonia & Europa & Mixto & Chile & Sur América & Mixto \\
\hline Lituania & Europa & Corporativo & \multirow{2}{*}{ Países no OECD } & & \\
\hline Luxemburgo & Europa & Corporativo & & & \\
\hline Holanda & Europa & Corporativo & Rusia & Europa & Corporativo \\
\hline Noruega & Europa & Democrático & Argentina & Sur América & Democrático \\
\hline Polonia & Europa & Democrático & Perú & Sur América & Democrático \\
\hline Portugal & Europa & Mixto & Brasil & Sur América & Corporativo \\
\hline Eslovaquia & Europa & Mixto & Colombia & Sur América & Mixto \\
\hline Eslovenia & Europa & Mixto & Sud África & África & Corporativo \\
\hline
\end{tabular}

Actualmente, los gobiernos universitarios capturan bastante interés académico, con énfasis en la forma en que enfrentan el nuevo entorno de diversidad de fuentes de financiamiento, globalización y competencia (ANAO, 2003). Los desafíos actuales de las universidades parecen centrarse en la búsqueda de mayor autonomía ante el Estado, mejores sistemas de rendición de cuentas (accountability) (Fielden 2008), responsabilidad institucional completa ante la sociedad, y sistemas de gobierno basados en prioridades estratégicas y en la gestión profesional (Paradeise et al., 2009 y Larsson, 2006). 


\section{Desempeño de las Universidades}

El desempeño de las universidades es bastante difícil de medir (Reyes, 2016). Algunas proxies utilizan una serie de indicadores de gestión y de resultados. En nuestro caso se buscan medidas que permitan una medición aproximada en base a información sencilla de recopilar y de validar. Para esto se consideraron medidas de la posición en un ranking internacional de las universidades, y también se utilizó una medida de desempeño económico de las universidades basadas en medidas de creación de Valor. Estas dos dimensiones, posición en rankings internacionales y resultados económicos, son los indicadores que aquí usamos como proxys de desempeño de estas instituciones.

\section{Hipótesis del estudio}

A partir de lo anteriormente expuesto, en este estudio nos centramos a analizar la relación entre el desempeño (académico y económico) de las universidades y medidas de gobierno universitario. Para esto formulamos las siguientes hipótesis: Hipótesis 1: "El desempeño académico de las universidades chilenas (posición y tendencia en el ranking) está asociada con la forma en que se eligen a sus rectores"; Hipótesis 2: "El desempeño académico de las universidades chilenas (posición y tendencia en el ranking) está asociado con el perfil de sus rectores"; Hipótesis 3: "Las universidades chilenas que votan para elegir al rector tienen mejor desempeño económico que las universidades en que el rector es designado externamente"; e Hipótesis 4: "El desempeño académico de las universidades chilenas (posición y tendencia en el ranking) está asociada con el tipo de propiedad de estas (estatal-privada)". La propiedad es una variable que caracteriza o tipifica a las universidades, y que por ello puede afectar la relación de los modelos de gobiernos con el desempeño.

\section{METODOLOGÍA}

Respecto del diseño de investigación, la recolección de los datos y su medición, la metodología se describe como sigue:

a) Hemos utilizado el Ranking Internacional SciMago (Scimago Institutions Rankings), que ofrece mediciones anuales de libre acceso para un gran número de universidades a través del mundo. Esto permite evaluar con una misma metodología la evolución en el ranking de cada universidad. Este ranking es muy dependiente del tamaño de las universidades, y se basa en tres factores: investigación (50\%), innovación (30\%) y sociedad (20\%). Se tiene la posición anual en el ranking mundial 2009-2018. SciMago usa ranking tipo 1223, y nosotros la hemos convertido al sistema 1224. También hemos estimado la tendencia (pendiente) usando MCO, como indicador del número de posiciones promedio anual que cada universidad ha subido o bajado (dy/dy) en el ranking mundial (sistema 1224). Con ello hemos generado tres categorías: Caída grande: pendiente<-90; Caída leve: pendiente entre -90 y 0; Mejora: pendiente $>=0$.

b) Caracterización de la universidad y su gobierno. Las universidades han sido caracterizadas como sigue: Por propiedad: Criterio 1: Estatal/Privada. Criterio 2: Estatal/Red-G9/Privada no Red-G9. Respecto del sistema de elección del rector, se responde la siguiente pregunta ¿los profesores de la universidad votan directamente por un candidato a rector? Sí/No. Se considera que hay votación directa cuando los profesores (o un claustro de éstos en conjunto con otros estamentos internos) lo hacen por un candidato, independiente que el candidato con mayoría (o la terna) deba ser ratificado o seleccionado por una autoridad superior o no (un ejemplo es sistema de la PUC de Valparaíso). Por otro lado, se considera que no hay votación directa cuando el rector es designado por alguna junta directiva externa a la universidad, o por un comité de búsqueda del rector (ejemplos son los sistemas de la PUC, UCN, UC Maule, UC Temuco, UCS Concepción).

Respecto del perfil del rector actual, se consideró lo siguiente: a) Experiencia en alta gerencia privada y/o universidades privadas: directivo con supervisión de: menos de 25 trabajadores / entre 25 y 200 trabajadores / 200 o más trabajadores. b) Respecto de la experiencia en alta gerencia pública y/o universitaria estatal: las categorías fueron: Sin experiencia directiva / Experiencia nivel regional (seremis, directores regionales de servicios públicos) o dentro de la misma universidad (decanos o rectores previos, secretarios generales, vicerrectores) / Experiencia nivel nacional (ministros de estado, embajadores, directores de servicios públicos). c) Respecto del Perfil científico de los rectores: Hemos usado el h-index del WoS (core collection) como medida de producción científica (Hirsch, 2005). Fuentes complementarias para identificar los nombres correctos de los rectores fueron ReserchID y Dataciencia de Conicyt (https://dataciencia.conicyt.cl). Se usó dos categorías de clasificación: $\mathrm{h}$-index $<4$ / h-index $>=4$.

Respecto del análisis de datos, para evaluar formalmente las hipótesis centrales utilizamos dos pruebas no paramétricas usuales de independencia condicional en tablas de contingencia bidireccionales: Pearson Chi2 y el Likelihood-ratio test (LR). La hipótesis nula en ambos casos es que filas y columnas de las tablas son independientes entre sí. En todos los casos se trabajó con un de nivel de significancia de 5\%. La estadística 
de Chi cuadrado se usa comúnmente para probar las relaciones entre variables categóricas. La ecuación (1) muestra que el estadístico Chi2 se basa en la diferencia entre lo que se observa en los datos y lo que se esperaría si realmente no hubiera relación entre las variables, es decir:

$$
x^{2}=\sum \frac{\left(f_{0}-f_{e}\right)^{2}}{f_{e}}
$$

donde fo es la frecuencia observada y fe la frecuencia esperada si no existiera relación entre las variables. Por otro lado, el estadístico de prueba de razón de verosimilitud (Likelihood-ratio test) tiene la forma indicada en la ecuación (2):

$$
\chi_{2}^{2}=2 \sum n_{i j} \ln \left(\frac{n_{i j}}{E_{i j}}\right)
$$

donde el estadístico se compara con la distribución Chi2 con (r-1) (c-1) grados de libertad. Esta estadística es similar a la estadística Chi2 de Pearson a menos que haya una o más frecuencias esperadas muy pequeñas en la tabla. Koehler y Larntz (1980) indican que el estadístico Chi2 suele ser más apropiado para tablas con frecuencias esperadas pequeñas. Sin embargo, la estadística de razón de verosimilitud tiene una propiedad aditiva que la hace más deseable en ciertos casos.

\section{RESULTADOS Y DISCUSIÓN}

La base de datos quedó constituida por 31 universidades chilenas (Tabla 3) que aparecen en el ranking Scimago. Varias de estas universidades ingresaron al ranking hace pocos años, y se tiene menos información de ellas. En la Tabla 3 puede observarse que la proporción de universidades que votan directamente por sus respectivos rectores es similar a las que no lo hacen: $\mathrm{No}=15(48.4 \%)$ versus $\mathrm{Si}=16(51.6 \%)$. Por otro lado, las universidades estatales en promedio tienen un EVA negativo (\$-3.492 millones de pesos chilenos), mientras las universidades privadas tienen EVA promedio positivo ( $\$ 5.080$ la Red-G9 y $\$ 2.255$ las no Red-G9). Respecto del perfil de los rectores actuales, la gran mayoría (71\%) tienen una experiencia regular o media en el sector público o universidades. Menos del $30 \%$ de ellos pertenece a la categoría de investigadores destacados, y menos del $10 \%$ tienen experiencia en el sector privado.

\section{Posiciones y Tendencias Recientes en el Ranking Internacional}

La Figura 1a muestra que las universidades privadas ocupan posiciones promedio levemente mejores (menor número indica lugar más alto en el ranking mundial) que las estatales. Sin embargo, al clasificar las universidades en tres grupos (Figura 1b), las mejor posicionadas son las de la Red-G9, mientras las privadas nuevas (no Red-G9) ocupan las posiciones menos favorables. Respecto de las tendencias recientes, la Figura 2 reporta la mediana del cambio de cada universidad en el ranking mundial 2009-2018 (valores negativos indican caídas en el ranking). Aquí puede observarse que globalmente todos los grupos de universidades chilenas presentan caídas, y que son las universidades estatales las que presentan las mayores caídas. Pero en realidad son las universidades privadas nuevas (no-G9) las que han tenido una evolución menos mala (Figura 2b), algo que no ha ocurrido por ejemplo en España (Casani et al., 2014). A partir de esto puede inferirse que, de mantenerse las tendencias, las posiciones actuales en el ranking (Figura 1) pueden cambiar sustantivamente.

Tabla 3. Universidades Incluidas en el Estudio. Características básicas en base al Ranking Scimago 2009-2018, Zuniga-Jara et al. (2018) y Zuniga-Jara et al. (2020).

\begin{tabular}{|c|l|c|c|c|c|c|}
\hline Num. & Institución & Tipo & Ranking Scimago & Tendencia & Votación & EVA 2017 \\
\hline & & & 2018 (sist.1224) & 2009-2018 & para rector & millones de CH\$ \\
\hline 1 & U. de Chile & Estatal & 507 & mejora & Sí & -27.072 \\
\hline 2 & Pontificia U. Católica de Chile & Red G9 & 701 & mejora & No & 28.446 \\
\hline 3 & U. de los Andes & Privada no G9 & 930 & mejora & No & -3.666 \\
\hline 4 & U. Técnica Federico Santa María & Red-G9 & 1158 & caída leve & Sí & -6.082 \\
\hline 5 & U. Andrés Bello & Privada no G9 & 1188 & mejora & No & 10.791 \\
\hline 6 & U. de Santiago de Chile & Estatal & 1382 & caída leve & Sí & -946 \\
\hline 7 & U. de Concepción & Red-G9 & 1510 & caída leve & Sí & 4.925 \\
\hline 8 & U. Austral de Chile & Red-G9 & 1756 & caída leve & Sí & -6.122 \\
\hline 9 & U. de Valparaíso & Estatal & 1831 & caída leve & Sí & -976 \\
\hline 10 & Pont. U. Católica de Valparaíso & Red-G9 & 2042 & caída leve & Sí & 12.926 \\
\hline 11 & U. de Talca & Estatal & 2092 & caída leve & Sí & -745 \\
\hline
\end{tabular}


Tabla 3. Continuación.

\begin{tabular}{|l|l|c|c|c|c|c|}
\hline Num. & Institución & Tipo & Ranking Scimago & Tendencia & Votación & EVA 2017 \\
\hline & & & 2018 (sist.1224) & $2009-2018$ & para rector & millones de CH\$ \\
\hline 12 & U. de la Frontera & Estatal & 2106 & caída leve & Sí & -2.125 \\
\hline 13 & U. de Antofagasta & Estatal & 2225 & mejora & Sí & -21 \\
\hline 14 & U. Diego Portales & Privada no G9 & 2323 & mejora & No & 1.018 \\
\hline 15 & U. Católica del Norte & Red-G9 & 2475 & caída leve & No & -2.794 \\
\hline 16 & U. del Desarrollo & Privada no G9 & 2612 & caída leve & No & 4.03 \\
\hline 17 & U. del Bio-Bio & Estatal & 2741 & caída grande & Sí & 231 \\
\hline 18 & U. Católica de Temuco & Red-G9 & 2741 & mejora & No & 6.287 \\
\hline 19 & U. Autónoma de Chile & Privada no G9 & 2800 & mejora & No & 5.931 \\
\hline 20 & U. de Tarapacá & Estatal & 2800 & caída grande & Sí & -2.517 \\
\hline 21 & U. Adolfo Ibáñez & Privada no G9 & 2858 & caída grande & No & 684 \\
\hline 22 & U. de La Serena & Estatal & 2872 & caída grande & Sí & -1.859 \\
\hline 23 & U. San Sebastián & Privada no G9 & 2986 & caída grande & No & 8.264 \\
\hline 24 & U. Católica del Maule & Red-G9 & 2998 & & No & 4.518 \\
\hline 25 & U. Santo Tomas, Chile & Privada no G9 & 3058 & caída grande & No & -1.472 \\
\hline 26 & U. Católica de la S. Concepción & Red-G9 & 3072 & & No & 3.617 \\
\hline 27 & U. Alberto Hurtado & Privada no G9 & 3126 & & No & -955 \\
\hline 28 & U. de Magallanes & Estatal & 3141 & & Sí & -3.672 \\
\hline 29 & U. de Los Lagos & Estatal & 3181 & & Sí & -962 \\
\hline 30 & U. Central de Chile & Privada no G9 & 3195 & & No & -2.076 \\
\hline 31 & U. de Playa Ancha & 3204 & caída grande & Sí & -1.238 \\
\hline & & & & \\
\hline
\end{tabular}

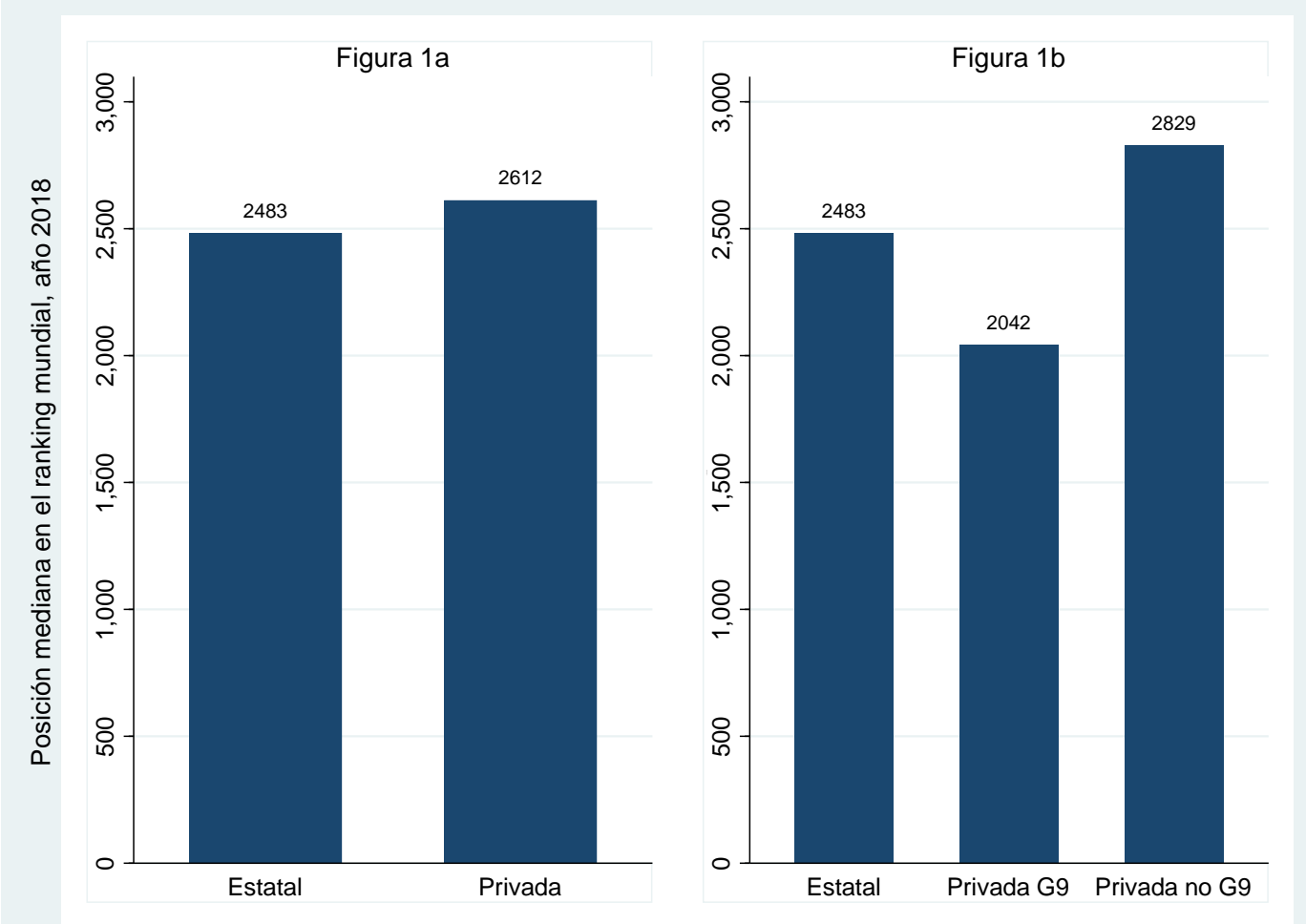

Fig. 1. Posiciones medianas en el ranking mundial de las universidades chilenas año 2018. Menor posición en el ranking es indicador de mejor desempeño.

\section{Hipótesis del Desempeño de las Universidades}

La Hipótesis 1 postula que el desempeño académico de las universidades (posición y tendencia en el ranking) está asociada con la forma en que se designan a los rectores (votación directa o no). Para evaluar esto, por una parte, la posición de las universidades en el ranking del año 2018 fue clasificada en cuatro categorías: menor a 1000 (las universidades mejor rankeadas), entre las posiciones 1000 y 2000, entre las posiciones 2000 y 3000, y posiciones mayores a 3000 (i.e., con ranking más bajo). Por otro lado, también se utilizó la 
tendencia en el ranking en el periodo 2009-2018. Los resultados basados en la Tabla 4a) sugieren que las universidades que eligen a sus rectores a través de votación directa, son las que registran proporcionalmente mayores caídas (tendencia) en el ranking (Pearson chi2(2) $=5,462, \operatorname{Pr}=0,065, y$ LR chi2(2) $=5,7304, \operatorname{Pr}=0,057$ ). Nuevamente no fue posible obtener conclusiones estadísticas para el caso de la posición en el ranking un año en particular. En resumen, este resultado sugiere que la Hipótesis 2 es verdadera, pero sólo para la tendencia a través de varios años.

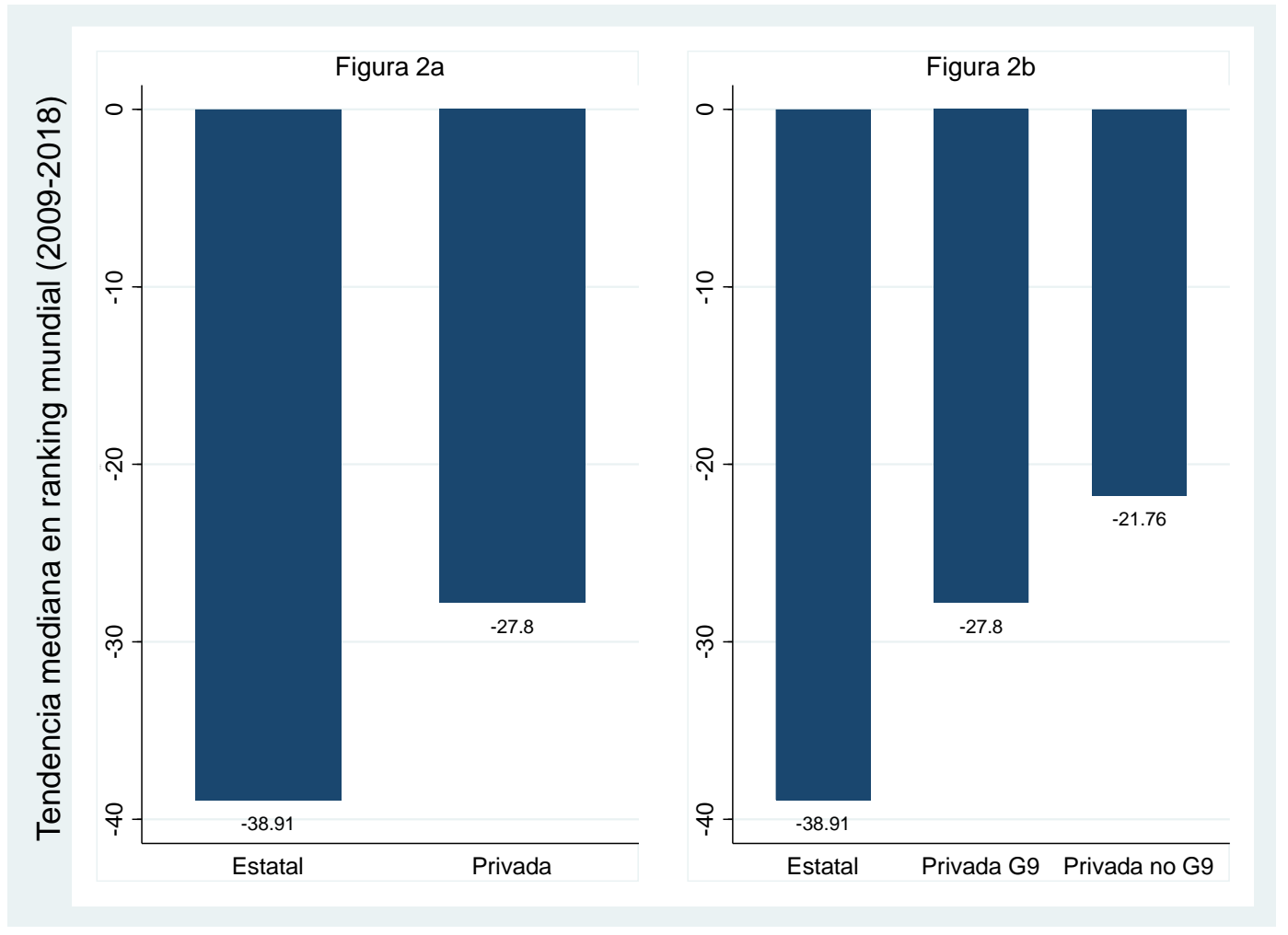

Fig. 2. Mediana de la tendencia (pendiente) en las posiciones en el ranking mundial de las universidades chilenas (2009-2018). Pendientes negativas indican un empeoramiento en el ranking a través del tiempo.

La Hipótesis 2 postula que el desempeño académico de las universidades (posición y tendencia en el ranking) está asociada con el perfil de sus rectores. En este caso, el perfil de los rectores como investigador destacado aparece vinculado solamente con la posición en el ranking el año 2018 (Pearson chi2(3)=6,247, P-value=0,10, y LR chi2(3)=7,817 y P-value=0,05). La distribución de frecuencias en la Tabla $4 b$ ) muestra que estadísticamente las universidades mejor rankeadas (privadas y estatales) tienden a designar rectores con un perfil de investigador destacado. En el caso del perfil relacionado con la experiencia en el sector privado y en el sector público del rector, ninguno de estos implicó una relación estadística con el desempeño de las universidades. En resumen, este resultado sugiere que la Hipótesis 3 es verdadera, pero sólo para el año 2018.

La Hipótesis 3 postula que el desempeño económico de las universidades está asociado a la forma en que designan a los rectores. La Tabla 4c) muestra cómo se relaciona el EVA (como indicador de desempeño económico), con la forma en que es designado el rector. El EVA fue organizado en cuatro categorías (en millones de pesos chilenos): muy negativo (menor a \$-10.000), negativo (entre \$-10.000 y \$0), positivo (entre $\$ 0$ y $\$ 10.000$ ), y muy positivo (mayor a $\$ 10.000$ ). En este caso la hipótesis no es rechazada estadísticamente con bastante claridad (Pearson chi2(3)=7,792, P-value $=0,051$, y LR chi2(3) $=8,519, P$-value $=0,036$ ). Esto sugiere una clara vinculación entre un resultado financiero positivo (creación de valor), con el hecho de que el rector sea designado por un tipo de comité de búsqueda. Por otro lado, el desempeño económico de la universidad no apareció vinculado estadísticamente ni a la experiencia en el sector privado o pública del rector, ni al hecho de ser investigador destacado o no.

Finalmente, la Hipótesis 4 postulaba que el desempeño académico (posición y tendencia en el ranking) de las universidades está asociada con el tipo de propiedad: estatal, privada G-9 y privada no G-9. Usando la distribución de frecuencias de la Tabla 4d) encontramos que la caída en los rankings (pendiente o tendencia en el periodo 2009-2018) está vinculada estadísticamente con el tipo de propiedad de las universidades (Pearson chi2(4)=7.259, P-value $=0.12$ y LR chi2(4)=9.315, P-value $=0.05)$. Por otro lado, no encontramos una relación estadística entre la posición en el ranking 2018 y la propiedad de las universidades. En resumen, 
este resultado sugiere que es posible asociar la tendencia en el ranking a través de varios años de las universidades con su propiedad, mientras que la posición puntual que tiene una universidad en un año, no proporciona suficiente información como para permitir esta asociación. A partir de esto, globalmente los resultados parecen apoyar el modelo corporativo de universidades, el cual predomina en las mejores universidades del mundo, en que se enfatiza la gestión orientada a los negocios (Hong, 2018), la internacionalización y la competencia (Dobbins y Knill, 2017).

Tabla 4. Contingencia para Variables con Relaciones Estadísticas Significativas. EVA en millones de pesos chilenos. La hipótesis nula es que filas y columnas de las tablas son independientes entre sí.

\begin{tabular}{|l|c|c|c|}
\hline Tabla 4a) & \multicolumn{3}{|c|}{ Forma de elección del rector } \\
\hline & No votan & Votan & 7 \\
\hline Caída grande en ranking 2009-2018 & 3 & 4 & 10 \\
\hline Caída leve en ranking 2009-2018 & 2 & 8 & 8 \\
\hline Mejora en ranking 2009-2018 & 6 & 2 & 25 \\
\hline Total & 11 & 14 & \\
\hline
\end{tabular}

\begin{tabular}{|l|c|c|c|}
\hline Tabla $4 \mathrm{~b})$ & \multicolumn{3}{|c|}{ Perfil del rector } \\
\hline & $\begin{array}{c}\text { No investigador } \\
\text { destacado }\end{array}$ & $\begin{array}{c}\text { Investigador } \\
\text { destacado }\end{array}$ & Total \\
\hline Ranking 2018<1000 & 1 & 2 & 3 \\
\hline Ranking 2018 entre 1000 y 2000 & 3 & 3 & 6 \\
\hline Ranking 2018 entre 2000 y 3000 & 11 & 4 & 15 \\
\hline Ranking 2018>=3000 & 7 & 0 & 7 \\
\hline Total & 22 & 9 & 31 \\
\hline
\end{tabular}

\begin{tabular}{|l|c|c|c|}
\hline Tabla 4c) & \multicolumn{3}{|c|}{ Forma de elegir al rector } \\
\hline & No votan & Votan & 1 \\
\hline Desempeño económico muy negativo & 0 & 1 & 17 \\
\hline Desempeño económico negativo & 5 & 12 & 10 \\
\hline Desempeño económico positivo & 8 & 2 & 3 \\
\hline Desempeño económico muy positivo & 2 & 1 & 31 \\
\hline Total & 15 & 16 & \\
\hline
\end{tabular}

\begin{tabular}{|l|c|c|c|c|}
\hline Tabla 4d) & \multicolumn{5}{|c|}{ Propiedad } \\
\hline Caída grande en ranking 2009-2018 & Estatal & Red-G9 & No Red-G9 & Total \\
\hline Caída leve en ranking 2009-2018 & 4 & 0 & 3 & 7 \\
\hline Mejora en ranking 2009-2018 & 4 & 5 & 1 & 10 \\
\hline Total & 2 & 2 & 4 & 8 \\
\hline
\end{tabular}

Cómo limitación de nuestro estudio, podemos señalar que se refiere sólo al caso chileno, y plantea correlaciones estadísticas y no causalidades (e.g. tipo Granger). Por ello, queda abierta la interrogante de si es el rector-investigador quien parece impulsar a la mejora de la universidad, o por el contrario, si debido a que la universidad está bien posicionada (y quiere mantener ese nivel), es que elige a rectores-investigadores.

\section{CONCLUSIONES}

A partir de los resultados obtenidos, se pueden extraer las siguientes conclusiones: 1) El desempeño de las universidades chilenas está asociado con la forma en que los rectores son designados. 2) El desempeño de las universidades chilenas está asociado con el perfil profesional de los rectores. 3) Los resultados globalmente sugieren que en Chile el sistema de gobierno corporativo universitario juega un papel importante en los resultados. 4) Existe una clara vinculación entre un resultado financiero positivo (creación de valor) de las universidades, con el hecho de que el rector sea designado por un tipo de comité de búsqueda (que no sea elegido democráticamente).

\section{REFERENCIAS}

Aghion, P., Dewatripont, M., y otros tres autores, The governance and performance of universities: evidence from Europe and the US., doi:10.1111/j.1468-0327.2009.00238.x, Economic Policy, 25(61), 7-59 (2010).

ANAO, Australian National Audit Office, Better Practice Guide: Public Sector Governance, Australian National Audit Office, Canberra, p.6. (2003).

Bernasconi, A., Is There a Latin American Model of the University? Comparative Education Review, Vol. 52, No. 1 (February 2008), pp. 27-52 (2008a). 
Bernasconi, A., La crisis del modelo latinoamericano de la universidad. En Brunner, J.J. y Peña, C. (2008): Reforma de la Educación Superior. Ed. Universidad Diego Portales, Santiago (2008b).

Bernasconi, A., La difícil tarea de clasificar universidades, doi: https://doi.org/10.31619/caledu.n25.254, Calidad en la Educación, (25), 81-96. (2018).

Bleiklie, I. y Kogan, M., Organization and Governance of Universities, Higher Education Policy, 2007, 20: 477-493 (2007).

Brunner, J.J., Gobernanza universitaria: tipología, dinámicas y tendencias, Revista de Educación, Vol. 355. Mayo-agosto 2011, pp. 137-159 (2011).

Brunner, J.J. y Peña, C., Reforma de la Educación Superior, Ed. Universidad Diego Portales, Santiago (2008).

Casani, F., De Filippo, D., García-Zorita, C., Sanz-Casado, E., Public versus private universities: Assessment of research performance; case study of the Spanish university system, https://doi.org/10.1093/reseval/rvt028, Research Evaluation, Vol 23(1) 2014, 48-61, (2014).

CGR, Financiamiento Fiscal a la Educación Superior, Contraloría General de la República de Chile, Informes anuales (2017).

Dobbins, M. y Knill, CH., Higher education governance in France, Germany, and Italy: Change and variation in the impact of transnational soft governance, doi: 10.1080/14494035.2017.1278868, Policy and Society, 36:1, 67-88, (2017).

Edwards, S., El deterioro de la Universidad de Chile, El Mercurio.com - Blogs. Diario El Mercurio, Chile, 05 de agosto (2018).

Frenken, K., Heimeriks, GJ., Hoekman, J., What drives university research performance? An analysis using the CWTS Leiden Ranking data, https://doi.org/10.1016/j.joi.2017.06.006, Journal of Informetrics. Volume 11, Issue 3, August 2017, Pages 859-872. (2017).

Fielden, J., Global trends in university governance, Education working paper series no. 9. Washington, DC: World Bank (2008).

Henze, R., Corporate Governance Perspectives, doi: 10.1080/13603101003779915, Policy and Practice in Higher Education, 14:3, 86-90, (2010).

Hirsch, J. E., An index to quantify an individual's scientific research output, doi:10.1073/pnas.0507655102, PNAS. 102 (46) 16569-72. (2005).

Hong, M., Public university governance in China and Australia: a comparative study, https://doi.org/10.1007/s10734-0180234-5, High Educ. Vol 76, Issue 4, pp 717-733. (2018).

Nahuelhual, J., Reforma a gobierno corporativo de universidades estatales surge en medio de debate sobre lucro y educación, La Tercera, edición del 21-agosto (2011).

Koehler, K.J. y K. Larntz, An Empirical Investigation of Goodness-of-Fit Statistics for Sparse Multinomials, doi: 10.2307/2287455, Journal of the American Statistical Association, Vol. 75, No. 370, pp. 336-344. (1980).

Larsson, A., Governing Bodies of Higher Education Institutions. Roles and responsibilities, OECD Seminar, Paris, 24-25 August (2006).

Ordorika, I., Elección de rector: panorama internacional, Revista de la educación superior, vol.44 no.175 México jul./sep. 2015. ISSN 0185-2760 (2015).

Paradeise C., Reale, E., Bleiklie, I., Ferlie, E. (Eds.), University Governance: Western European Comparative Perspectives, doi: 10.1007/978-1-4020-9515-3, Springer Netherlands, (2009).

Reyes, C., Medición de la calidad universitaria en Chile: la influencia de los rankings, http://dx.doi.org/10.4067/S071845652016000100007, Calidad en la educación. №44, julio. (2016).

Rupp, DE., Batz, C., y otros tres autores, Competencies for State College and University Presidents, American Association of State Colleges and Universities (2016).

Torres, E., Gobierno universitario en entornos de debate social en Chile. Observaciones desde la Teoría de sistemas, http://dx.doi.org/10.4067/S0718-65682016000100030, Polis vol.15 no.43 Santiago abr. 2016. (2016).

Rodríguez, D., Trato preferente y captura de las universidades del Estado, El Mostrador, 15 marzo (2016).

UWN, Business calls for rectors to be elected by boards, University World News by Jan Petter Myklebust. 23 September 2016 Issue No:429m (2016).

Zuniga-Jara, S., Barraza-Carvajal, J., Sanhueza-Muñoz, N., Soza-Amigo, S., Midiendo la Creación de Valor en una Universidad, http://dx.doi.org/10.4067/S0718-50062018000300087, Formación Universitaria. vol.11 no.3 (2018).

Zuniga-Jara, S., Sjoberg-Tapia, O., Rojas-Guerra, J. y Valenzuela-Cortes, T., ¿Crean Valor las Universidades Chilenas?, Por aparecer en Formación Universitaria, vol. 13 no. 6 (2020). 
